\title{
Actualización en el manejo anestésico del paciente obeso. ¿Lo sabemos todo?
}

Artículo original: Bazurro S, Ball L, Pelosi P. Perioperative management of obese pacient. Current opinion in Critical Care 2018, 24 (6): 560-567. (

Sánchez Quirós B, Pérez Herrero MA.

\section{Hospital Clínico Universitario de Valladolid.}

\section{Resumen}

La obesidad creciente en nuestro medio es un factor de riesgo para la aparición de complicaciones perioperatorias. Un manejo perioperatorio individualizado, teniendo en cuenta la idiosincrasia de estos pacientes es esencial para mejorar los resultados y la seguridad del procedimiento quirúrgico. Un manejo anestésico adecuado puede cambiar sustancialmente el desenlace vital de estos pacientes.

\section{Introducción}

La obesidad creciente en nuestro medio es un factor de riesgo para la aparición de complicaciones perioperatorias. Un manejo perioperatorio individualizado, teniendo en cuenta la idiosincrasia de estos pacientes es esencial para mejorar los resultados y la seguridad del procedimiento quirúrgico. Un manejo anestesico adecuado puede cambiar sustancialmente el desenlace vital de estos pacientes.

La obesidad no es sólo un problema de salud pública, sino un reto para el anestesiólogo, al que nos enfrentamos con frecuencia creciente: presenta una prevalencia del $13 \%$ de la población mundial (el $39 \%$ sobrepeso) ${ }^{1}$. En España, la prevalencia de obesidad se ha duplicado en los últimos años ${ }^{2}$.

Los avances anestésicos han posibilitado la realización de procedimientos quirúrgicos en pacientes de alto riesgo, incluso en régimen ambulatorio. La identificación de los pacientes de alto riesgo facilita la indicación y optimización perioperatoria de los mismos. La obesidad es uno de los factores de riesgo independientes de morbimortalidad quirúrgica, junto a la edad, estado físico (ASA), apnea obstructiva del sueño y la fragilidad.

Las características anatómicas y fisiológicas de los pacientes obesos presentan una serie de comorbilidades asociadas, con frecuencia infradiagnosticados, entre las que destaca el síndrome metabólico, las complicaciones respiratorias, las cardiopatías y el estado protrombótico.

La sospecha y optimización de éstas reduce significativamente la morbimortalidad perioperatoria ${ }^{3}$.

En este estudio, nos propusimos revisar la evidencia actual sobre el manejo anestésico perioperatorio y la evaluación de situaciones de riesgo, con el objetivo de establecer las mejores prácticas anestésicas en este grupo de pacientes. 


\section{Material y Métodos}

A pesar del bajo número de guías clínicas para el manejo perioperatorio del paciente obeso, el artículo toma como base las publicadas en Reino Unido, Irlanda e Italia. Por nuestra parte, realizamos una búsqueda para ayudarnos en el comentario del artículo utilizando los términos "anaesthesia" \& "obesity" \& "management" en las bases de datos MEDLINE, COCHRANE y EMBASE en octubre de 2018. Se encontraron 819 artículos, de los cuales se seleccionaron 288 publicados en los últimos cinco años sobre pacientes adultos no obstétricos.

\section{Resultados}

La anestesia y relajación muscular altera la fisiopatología respiratoria del paciente obeso en cuanto a la funcionalidad pulmonar y el intercambio de gases. La reducción del tamaño alveolar y la formación de atelectasias provoca la disminución de la capacidad residual funcional y de la reserva espiratoria; incluso el volumen pulmonar al final de la espiración puede ser menor que el volumen residual.

Esto, unido a la alteración de la relación ventilación/perfusión y a shunt pulmonar, dificulta el intercambio de gases, y en especial de la oxigenación.

La mecánica respiratoria se caracteriza por una reducción de la complianza y un aumento de la resistencia respiratoria, que se agrava en caso de aumento de la presión abdominal. Estos cambios se mantienen incluso dos ó tres días después de la intervención. Para evitarlo se recomienda asociar PEEP mayores de $10 \mathrm{~cm}$ de $\mathrm{H} 2 \mathrm{O}$ (generalmente de 18-20 cmH2O) a maniobras de reclutamiento alveolar a presiones de $55 \mathrm{~cm}$ de $\mathrm{H} 2 \mathrm{O}$ para evitar atelectasias; sin embargo, no hay evidencia de los resultados a largo plazo de esta estrategia ventilatoria. Se destaca la importancia de predecir el riesgo perioperatorio (las escalas más utilizadas son las OS-MRS, STPOPBang, ARISCAT, y LAS VEGAS) y así optimizar al paciente de forma multidisciplinar. Si bien la obesidad no es factor de riesgo independiente de complicación respiratoria, algunas características asociadas sí lo son como: 1-saturación arterial de oxígeno basal menor de 93\%. 2-obesidad mórbida (IMC $>50 \mathrm{~kg} / \mathrm{m}^{2}$ ). 3- SAOS o síndrome obesidad-hipoventilación. 4- las necesidades de ventilación no invasiva o monitorización cardiorrespiratoria.

El mal control de la glucemia y complicaciones intraoperatorias sangrado, procesos cardiorrespiratorios, y accidentes quirúrgicos- son también factores de riesgo de complicaciones que requieran ingreso en Unidad de críticos.

La mayoría de las escalas predictivas de intubación difícil incluyen la obesidad como factor de riesgo, especialmente cuando la circunferencia cervical es mayor de $41 \mathrm{~cm}$ en mujeres y de $43 \mathrm{~cm}$ en hombres o ratio cintura/cadera de 0,8 en hombres o 0,9 en mujeres. Ver tabla I.

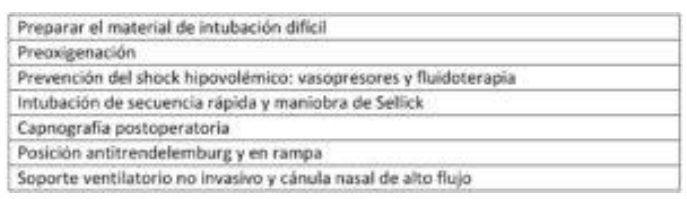

TABLA I. Recomendaciones para intubación del enfermo obeso

En cuanto a la optimización intraoperatoria, aunque sin evidencia suficiente, se recomienda anestesia libre de opioides, junto a la utilización de halogenados y de agentes de reciente descripción como la dexmedetomidina, con el fin de minimizar la respuesta inflamatoria asociada al acto anestésicoquirúrgico.

El artículo comentado sólo describe la fisiopatología del paciente obeso, con el 
fin de optimizar el manejo de la vía aérea y de la ventilación perioperatoria en función de la estratificación del riesgo de estos pacientes. Sin embargo, las características fisiopatológicas de la obesidad afectan a otros sistemas que deben tenerse en cuenta en el manejo anestésico de estos pacientes.

\section{Discusión}

Antes de seguir adelante con el tema, conviene recordar las definiciones de obesidad y sobrepeso. La obesidad es una enfermedad crónica $\mathrm{y}$ multifactorial, caracterizada por un exceso de tejido adiposo que afecta a la salud física y mental y reduce la expectativa de vida. ${ }^{4}$ Ya que la medida del contenido de grasa corporal es difícil y requiere de la realización de pruebas de imagen como la tomografía o resonancia magnética, se utiliza el concepto de peso ideal (IBW), que se calcula con la siguiente fórmula de la Figura 1.

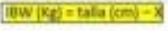

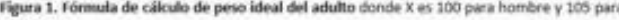

Figura 1. Fórmula de cálculo de peso ideal del adulto donde $\mathrm{X}$ es 100 para hombre y 105 para mujeres.

El Índice de Masa Corporal (IMC) es la relación entre peso y talla y se calcula como aparece en la figura 2 :

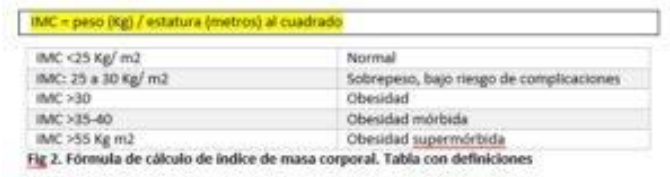

Fig 2. Fórmula de cálculo de índice de masa corporal. Tabla con definiciones

El paciente obeso presenta una serie de particularidades que deben evaluarse, prevenirse y tenerse en cuenta en el manejo anestésico perioperatorio. A continuación, resumimos por aparatos las complicaciones y recomendaciones a tener en cuenta, centrándonos en el síndrome metabólico y el aparato cardiocirculatorio y respiratorio, por ser estos los que más desenlaces adversos provocan:

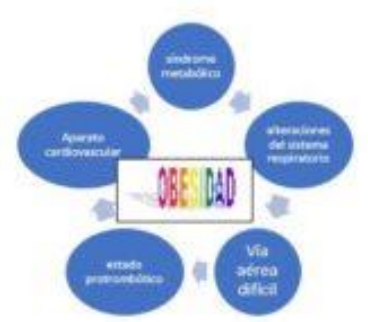

Figura 3. Aparatos afectados en la obesidad con implicaciones anestésico-quirúrgicas.

En el artículo comentado se recomienda tener prevista intubación difícil, y una monitorización hemodinámica continua, y ventilación mecánica protectora. En el postoperatorio, la colocación correcta, movilización precoz y la fisioterapia. No concreta el grado de obesidad a que se refiere y omite otros aparatos que pueden influir en el manejo anestésicoquirúrgico de estos pacientes, que revisamos y describimos a continuación.

\section{Síndrome metabólico}

Los pacientes obesos tienen mayor prevalencia de síndrome metabólico (hipercolesterolemia, hipertensión, obesidad central y resistencia a la insulina) que la población general, bien de forma parcial o completa. La obesidad troncular predispone a resistencia a la insulina y a diabetes mellitus. La respuesta catabólica a la cirugía puede requerir insulinoterapia para el control de la glucemia, pues se ha demostrado que un buen control glucémico perioperatorio mejora el pronóstico ${ }^{3}$ presentando correlación con menor incidencia de infecciones y de enfermedad coronaria.

\section{Dosificación de fármacos}

Hay controversia en cuanto a la titulación de propofol en función del 
peso real o de la masa magra; pero es indiscutible una administración cuidadosa para evitar complicaciones de obstrucción de vía aérea, hipotensión o disminución del gasto cardiaco. Las dosis de relajantes musculares y de sugammadex debe ajustarse al peso real.

\section{Aparato respiratorio}

Los pacientes obesos presentan mayor trabajo respiratorio, debido a las resistencias respiratorias altas (sobre todo en caso de hipoxemia e hipercapnia), la presión abdominal elevada y la reducción de la compliance pulmonar. Por ello se deben evitar la ventilación espontánea y la posición de litotomía o Trendelemburg, pues se asocia a riesgo de hipoventilación, hipercapnia secundaria y fallo cardiaco derecho.

Por otra parte, el incremento en las demandas metabólicas y demanda de oxígeno, unido a un intercambio de gases defectuoso, provoca que los episodios de desaturación sean más rápidos y frecuentes que en condiciones normales. El tiempo seguro de apnea (entre la relajación muscular y la caída de saturación de oxihemoglobina ( $\mathrm{SaO} 2)$ a niveles críticos) es menor (2-3 minutos) que en normopeso (8-10 minutos). Por ello se recomienda una preoxigenación de más de 3 minutos de duración y estrategias de ventilación protectora $^{5}$, como las resumidas en la tabla II. También se ha utilizado el denominado THRIVE (transnasal humified rapid-insuflation of ventilatory exchange) con $70 \mathrm{~L} / \mathrm{min}$ de oxígeno humidificado para la preoxigenación $\mathrm{y}$ durante inducción y relajación muscular. A estas medidas hay que añadir que el gradiente de presión entre la presión plateau y la PEEP debe ser menor de $16 \mathrm{~cm}$ de $\mathrm{H} 2 \mathrm{O}$ en el paciente obeso (de 13 en el paciente con normopeso).

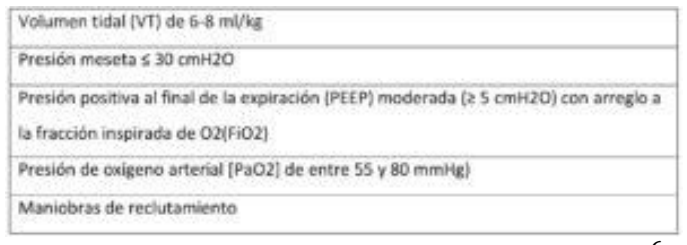

Tabla II. Estrategias de ventilación protectora. ${ }^{6}$

La apnea/hipopnea del sueño $(20 \%$ de pacientes con IMC $>35$, diagnosticado con polisomnografía y pruebas funcionales) constituyen un factor independiente de morbimortalidad perioperatoria, pues se asocia a hipoxia, hipercapnia, hipertensión pulmonar $\mathrm{y}$ arterial y arritmias. La hipoxemia crónica provoca policitemia secundaria, mayor riesgo de enfermedad isquémica coronaria y cerebro vascular. Finalmente, la vasoconstricción pulmonar hipóxica provoca fallo de ventrículo derecho.

La valoración preanestésica de la vía aérea es imprescindible en todos los pacientes, pero en el paciente obeso debe realizarse teniendo en cuenta la elevada incidencia de ventilación e intubación difícil (hasta del 13\%), especialmente cuando la circunferencia cervical es mayor de $46 \mathrm{~cm}^{7}$ Se debe estudiar los parámetros clínicos descritos en la tabla III, teniendo previsto la intubación bajo fibroscopia y una cricotiroidotomía de urgencia en caso necesario. ${ }^{5}$

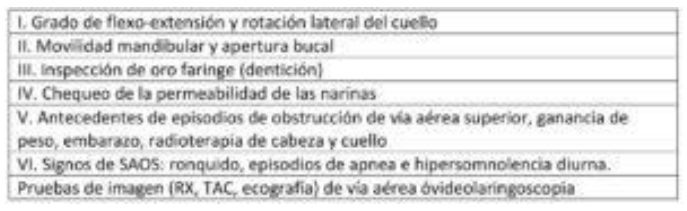

Tabla III. Evaluación de vía aérea

En aquellos pacientes con mayor posibilidad de complicaciones respiratorias, es aconsejable una anestesia libre o ahorradora de opioides, donde las técnicas locorregionales y estrategias como la perfusión intravenosa de dexmedetomidina. 7 Las figuras 4 y 5 describen las recomendaciones para el manejo 
perioperatorio y el postoperatorio inmediato de estos pacientes.

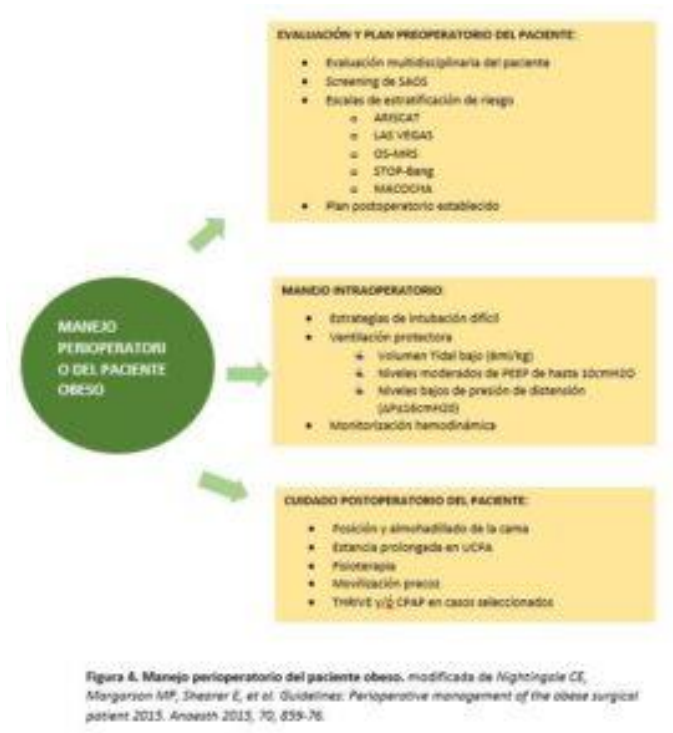

Figura 4. Manejo perioperatorio del paciente obeso. modificada de Nightingale CE, Margarson MP, Shearer E, et al. Guidelines: Perioperative management of the obese surgical patient 2015. Anaesth 2015, 70, 859-76.

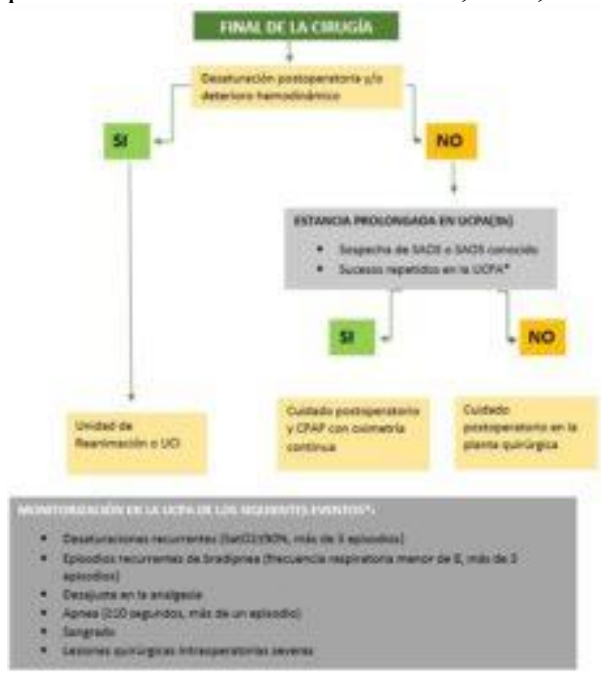

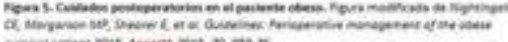

Figura 5. Cuidados postoperatorios en el paciente obeso. Figura modificada de Nightingale CE, Margarson MP, Shearer E, et al. Guidelines: Perioperative management of the obese surgical patient 2015. Anaesth 2015, 70, 859-76.

\section{$\underline{\text { 4. Aparato cardiocirculatorio }}$}

Los pacientes con IMC elevado presentan un riesgo elevado de arritmias, siendo la fibrilación auricular la más frecuente, por varias causas: disfunción del nodo sinusal, infiltración grasa del sistema de conducción, hipertrofia, hipoxemia, diuréticos (secundario a hipocalemia). Se ha descrito una correlación positiva entre obesidad y riesgo de hipertensión, enfermedad coronaria, y enfermedades cerebrovasculares ${ }^{3}$.

Por otra parte, el aumento de masa corporal provoca un aumento de la demanda metabólica y conduce a un aumento del gasto cardiaco que se ha calculado como un $20-30 \mathrm{ml} / \mathrm{kg}$ de exceso de grasa. La vasoconstricción pulmonar hipóxica puede evolucionar a hipertensión pulmonar crónica y fallo del ventrículo derecho. La hiperinsulinemia característica de la obesidad, puede activar el sistema nervioso y causar retención de sodio.

La valoración clínica preoperatoria del paciente obeso requiere de pruebas complementarias: electrocardiograma (de bajo voltaje, para estimar con precisión la gravedad de la hipertrofia ventricular), pruebas de imagen (radiografía torácica, ecocardiografía) y ergometría. Un aumento de peso en el preoperatorio puede indicar empeoramiento de la función cardiaca.

En cuanto a las consideraciones a tener en cuenta durante la anestesia, hay que destacar que la disfunción ventricular aparece asociada a la alteración respiratoria, pero su gravedad puede infraestimarse. La disminución perioperatoria del gasto cardiaco puede deberse a varias razones: disfunción ventricular diastólica izquierda por infusión rápida de líquidos $\mathrm{o}$ administración de fármacos con inotropismo negativo (inducción anestésica), o hipertensión pulmonar secundaria a hipoxia o hipercapnia (intubación orotraqueal). Por ello debe monitorizarse la presión arterial y el índice cardiaco de forma continua. 


\section{Hígado}

Los pacientes obesos suelen presentar hepatopatía tanto morfológica como funcional, como esteatosis, infiltración de mononucleares y neutrófilos, y fibrosis. El obeso grave suele presentar resistencia a la insulina. A veces se ha demostrado elevación de las transaminasas, fosfatasa alcalina, bilirrubina, y retención anómala de bromosulftaleína, sin relación con el grado de afectación funcional. Entre las recomendaciones anestésicas, se deberán evitar episodios de hipoxemia e hipotensión.

\section{Aparato Gastrointestinal}

A pesar de existir controversia en cuanto a la existencia de vaciamiento gástrico rápido y un volumen gástrico mayor en los obesos, se recomiendan medidas preventivas de la aspiración gástrica (ver tabla IV).

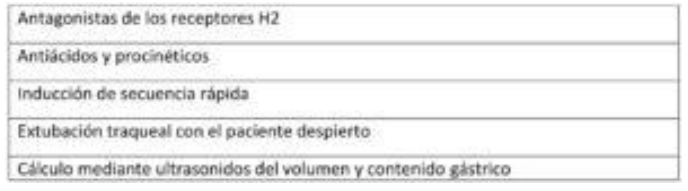

Tabla IV. Medidas de profilaxis de aspiración gástrica

\section{Estado protrombótico}

La obesidad es un estado procoagulante, sobre todo en pacientes femeninos (riesgo diez veces superior). Por ello, se deben realizar medidas de profilaxis antitrombótica antes, durante y después de la intervención en función del tipo de cirugía, el IMC y la comorbilidad del enfermo. Debemos considerar la posibilidad no solo de trombosis venosa profunda (TVP), sino también de isquemia mesentérica incluso más allá de dos semanas de cirugía ${ }^{8}$. Una de las principales medidas de profilaxis es limitar la duración de la anestesia, que depende claramente de la complejidad y duración de la cirugía, así como de la experiencia del cirujano ${ }^{5}$. $\underline{\text { 8. Cirugía con ingreso versus cirugía }}$ ambulatoria

La cirugía ambulatoria está indicada en caso de procedimientos menos invasivos y de menor duración, donde es posible el control del dolor y la recuperación integral precoz. Los protocolos de rehabilitación multimodal intensificada han mejorado los resultados quirúrgicos en todos los pacientes, pero especialmente en los obesos. Asimismo, el control perioperatorio e individualizado de las comorbilidades asociadas a la obesidad, en especial el SAOS, junto a un plan analgésico multimodal libre o ahorrador de opioides, donde las técnicas locorregionales tienen un papel fundamental, posibilitan la inclusión de este tipo de pacientes en programas de cirugía ambulatoria ${ }^{9}$.

Una vez revisada la guía de manejo perioperatorio del paciente obeso hemos podido observar que los resultados obtenidos en el manejo de la vía aérea, ventilación y complicaciones respiratorias postoperatorias, coinciden con la literatura revisada. Sin embargo, a pesar de ser este el campo que más morbimortalidad produce, es importante resaltar que hemos encontrado actualizaciones importantes en otras áreas. Estas medidas mejoran sustancialmente el resultado postquirúrgico y complementan a las guías actuales. La evaluación preanestésica completa, utilizando un buen screening de SAOS, pero también de patología cardiológica subyacente, síndrome metabólico y posibilidad de fenómenos tromboembólicos, no implantada actualmente en todos nuestros hospitales, disminuye la aparición de fenómenos adversos.

Es de especial interés ante la exponencial aparición de sujetos con IMC elevado en el ámbito quirúrgico, la formación de los profesionales en IOT 
con el paciente despierto, debido a la aumentada prevalencia de vía aérea difícil en estos pacientes. Hemos encontrado evidencias bibliográficas de sedación con dexmedetomidina para estos casos.

Por otro lado, parece necesario la protocolización en la inclusión del paciente obeso en la cirugía ambulatoria, aunque en este punto existen punto existen algunas hemos observado algunas debilidades, tales como la controversia entre la dificultad para las técnicas regionales que presentan estos pacientes y la desventaja que supone una anestesia general.

Resulta de especial interés individualizar la analgesia en estos pacientes, reduciendo los opioides y utilizando una analgesia multimodal, que podría ser una línea clave a revisar en próximas publicaciones.

\section{Conclusiones}

Todavía existen controversias en el manejo anestésico del paciente obeso, no sólo en cuanto a las indicaciones de la cirugía, sino también en cuanto al régimen idóneo de la misma (ambulatorio o con ingreso). Los pacientes obesos sufren mayor riesgo de complicaciones perioperatorias, generalmente respiratorias $y$ cardiovasculares. La optimización y la utilización de técnicas analgésicas y anestésicas multimodales, preferentemente locorregionales frente a general, individualizadas y libre $\mathrm{o}$ ahorradora de opioides perioperatoria es fundamental.

La evaluación preanestésica debe ser lo más exhaustiva posible, recomendándose profilaxis de tromboembolismo, aspiración gástrica e infección postoperatoria, siempre teniendo en cuenta la existencia de intubación y ventilación difícil; así como el riesgo de aspiración gástrica.

Hay que tener presente las alteraciones farmacocinéticas de estos pacientes, que requieren monitorización clínica y de la concentración plasmática de fármacos, en especial de aquellos con índices terapéuticos bajos (aminofilina, aminoglucósidos o digoxina). Los fármacos lipofílicos (tiopental, benzodiacepinas o inhalatorios) presentan un volumen de distribución elevado, y sus efectos persisten más de lo habitual.

En cuanto al manejo intraoperatorio las estrategias de preoxigenación $\mathrm{y}$ ventilación protectora son esenciales para disminuir las frecuentes complicaciones respiratorias que suelen desarrollar. Se recomienda la monitorización continua de presión arterial (invasiva o con manguito del tamaño adecuado) e índice cardíaco (pues la disminución del gasto cardiaco es frecuente); pulsioximetría, electrocardiografía, capnografía y neuroestimulación. La insulinoterapia ajustada a la monitorización estricta de glucemia ha demostrado disminuir la incidencia de infecciones y la enfermedad coronaria perioperatoria.

La utilización de ultrasonidos cobra aquí un papel primordial, tanto como herramienta para evaluación de la vía aérea, como para determinar el volumen de la cámara gástrica10, facilitar la canalización de vías venosas y arteriales, así como la realización de bloqueos nerviosos locorregionales.

Otras medidas recomendadas en el postoperatorio del paciente obeso son la vigilancia intensiva (evitando desaturaciones y apneas frecuentes y fallos de la analgesia) de mayor duración, fisioterapia y movilización precoz, y especial cuidado en la 
posición y almohadillado de las zonas de apoyo.

El control perioperatorio e individualizado de las comorbilidades asociadas a la obesidad (SAOS), junto a un plan analgésico multimodal libre o ahorrador de opioides, y la aplicación de protocolos de rehabilitación multimodal intensificada, han posibilitado la programación de procedimientos quirúrgicos en régimen de cirugía mayor ambulatoria.

\section{Bibliografía}

1.- OMS, informe 16 febrero de 2018. http://apps.who.int/mediacentre/factsheets /fs311/es/index.html

2.- Estrategia NAOS Ministerio de Sanidad y Consumo. www.aecosan.msssi.gob.es/AECOS AN/docs/documentos/nutricion/estrategianaos.p $\underline{\mathrm{df}}$

3.- Nightingale CE, Margarson MP, Shearer E, Redman JW, Lucas DN et al. Guidelines: Perioperative management of the obese surgical patient 2015. Anaesth 2015; 70, 859-76. ( $\underline{\mathrm{HTML}}$ ) ( $\underline{\mathrm{PDF}})$

4.- Adams JP, Murphy PG. Obesity in anaesthesia and intensive care, endocrine and metabolic disorders in anaesthesia and intensive

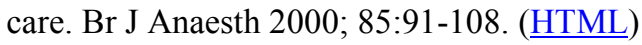

5.- Fyneface-Ogan S, Aqbam DS, Numbere C. Anaesthesic management of a super morbidly obese patiente for total abdominal hysterectomy: a few lessons to learn. African Health Sciences 2012; 12: 181-5. (HTML) (PDF)
6.- O'Gara B, Talmor D. Perioperative lung protective ventilation BMJ 2018; 10: 362:k3030. doi: 10.1136/bmj.k3030. (PubMed)

7.- Gaszynski T., Gaszynska E., Szewwczyk T. Dexmedetomidine for awake intubation and a opioid-free general anesthesia in a superobese patient with suspected difficult intubation. Drug Design, Development and Therapy 2014; 8: 909-12. (PubMed) ( $\underline{\text { HTML) (PDF) }}$

8.- Liew NC., Alemany GV., Angchaisuksiri P, Bang SM, Choi $G$ et al. Asian venous thromboembolism guidelines: updated recommendations for the prevention of venous thromboembolism. Int Angiol 2017; 36: 1-20. (PubMed)

9.- Walsh MT. Improving outcomes in ambulatory anesthesia by identifying high risk patients. Anestesiol 2018; 31: 659-66. (PubMed)

10.- Haskins SC, Kruisselbrink R, Boublik J, $\mathrm{Wu} \mathrm{CL}$, Perlas A. Gastric Ultrasound for the Regional Anesthesiologist and Pain Specialist. Reg Anesth Pain Med 2018; 43: 689-98. (PubMed)

Correspondencia al autor

Belén Sánchez Quirós

belensq93@gmail.com

MIR Anestesiología y Reanimación

Hospital Clínico Universitario de Valladolid.

Aceptado para el blog en marzo de 2019 